\title{
PACTOS EDUCATIVOS: DIFÍCILES PERO NECESARIOS
}

\author{
PACTOS EDUCATIVOS: \\ DIFÍCEIS, MAS NECESSÁRIOS \\ EDUCATIVE AGREEMENTS: \\ DIFFICULT BUT NECESSARY \\ PACTES ÉDUCATIFS: \\ DIFFICILES, MAIS NÉCESSAIRES
}

Juan Carlos Tedesco *

\section{RESUMEN}

En el documento se abordan uno de los temas más importantes de la agenda educativa contemporánea: los pactos educativos. Estos pueden considerar el producto deliberado de la negociación entre los diferentes actores sociales para enfrentar las tendencias a la fragmentación, la ruptura y la exclusión, tanto de los sectores más pobres de la población como de los sectores que ocupan la cúpula de la estructura social. El documento es dividido en función de tres palabras-clave: la necesidad de construir pactos que garanticen la continuidad de las politicas educativas; la dificultad para hacerlo; la posibilidad de lograr el objetivo: plantear la construcción de sociedades justas e cohesionadas.

Palabras-clave: Pacto educativo. Pacto social. Alianza educativa. Innovación en proyectos educativos. Concertación educativa. Politicas educativas.

* Director do Instituto Internacional de Planeamiento de la Educación - IIPE - Unesco, Sede Regional de Buenos Aires, Argentina (E-mail: j.tedesco@iipe-buenosaires.org.ar). 


\section{INTRODUCCIÓN}

Este documento está preparado para contribuir a los debates que tendrán lugar en el marco de las Jornadas que organiza la Fundación Santillana en Buenos Aires, sobre uno de los temas más importantes de la agenda educativa contemporánea: los pactos educativos. Este tema ya tiene cierta historia en las prácticas políticas utilizadas tanto en el plano nacional como internacional. Algunos autores se han ocupado de destacar las características de los pactos y de las alianzas educativas generadas a lo largo de la historia de los países latinoamericanos, así como de sus conflictos y sus rupturas (BRASLAVSKY, 1995, CASSASUS, 1995). Los pactos del pasado, sin embargo, eran productos más "orgánicos", asociados a alianzas de hecho entre diferentes sectores y actores sociales de una estructura social caracterizada por la presencia de proyectos que, en algunos casos exitosamente y en otros no, tenían fuertes características inclusivas. Los nuevos pactos, en cambio, son - o pretenden ser el producto deliberado, intencional, de la negociación entre esos diferentes actores sociales, para enfrentar las tendencias a la fragmentación, la ruptura y la exclusión tanto de los sectores más pobres de la población como de los sectores que ocupan la cúpula de la estructura social. Giddens (1999) llamó la atención, con acierto, sobre la existencia de procesos de des-afiliación en los dos extremos de la estructura social. En la base del sistema se producen fenómenos de expulsión mientras que en la cúpula se ponen de manifiesto conductas de auto-exclusión y de des-responsabilización.

En este sentido, es interesante recordar algunos hechos de la historia reciente. Hace poco más de diez años, en el mes de noviembre de 1993 y en la ciudad de Buenos Aires, cuatro instituciones (la FLACSO, la Fundación Concretar, la Fundación Ford y la OREALC UNESCO) con el auspicio del Ministerio de Educación de la República Argentina, organizaron un seminario internacional sobre concertación de políticas educativas que permitió discutir tanto los diferentes marcos teóricos acerca de esta problemática como las experiencias concretas que los países de América Latina estaban desarollando en esos momentos. Siete países (Argentina, Chile, Ecuador, Brasil, México, República Dominicana y Perú) presentaron análisis de sus experiencias y no es casual que los coordinadores del seminario (uno de ellos el actual Ministro de Educación de la República Argentina) titularan el libro con el interrogante acerca de la posibilidad de concertar políticas educativas (FLACSO, 1995).

Después de más de una década de la realización de ese seminario, no sólo el tema vuelve a ocupar un lugar importante en la agenda de discusiones académicas y políticas sino que el interrogante sigue vigente. $\mathrm{Al}$ respecto, es pertinente recordar que la O.E.I. dedicó el $\mathrm{n}^{\circ} 34$ de la Revista Iberoamericana de Educación correspondiente a los meses de enero-abril de 2004 al tema de los pactos educativos y que el artículo que introduce los materiales dedicados a discutir este tema también adopta la forma de una pregunta que, en definitiva, es la misma que se formularon los participantes del seminario de 1993. La diferencia, sin embargo, es que ahora tenemos la experiencia de haber intentado pactar y comprobamos que las dificultades son mucho más fuertes de lo que nos imaginábamos hace una década (TEDESCO, 2004).

En estos tres eventos - el Seminario de 1993, el número de la revista de la O.E.I. y estas Jornadas de la Fundación Santillana - se utilizan las tres palabras claves que sirven para entender el problema que nos ocupa. En el primer momento la pregunta giraba alrededor 
de la posibilidad de pactar; en el segundo el acento se trasladó hacia el análisis de la dificultad para hacerlo y ahora, en este encuentro, el foco se concentra en la necesidad de lograr un pacto educativo. Una síntesis de la posición que orienta este texto es invertir la secuencia de los tres términos mencionados y sostener que si el pacto es necesario, a pesar de las dificultades, tiene que ser posible.

Para sostener esta posición, hemos dividido el documento en función de las tres categorías mencionadas. En la primera parte trataremos de argumentar acerca de la necesidad que existe actualmente en nuestros países de construir pactos que garanticen la continuidad de las políticas educativas. En la segunda mostraremos las dificultades para el logro de este objetivo y en la última parte intentaremos mostrar que, a pesar de esas dificultades, el objetivo es posible. El texto recoge hipótesis y reflexiones que ya fueron expuestas en trabajos anteriores (TEDESCO, 1995). En esta versión, sin embargo, el objetivo fundamental es resumir lo que sabemos sobre este punto, postular algunas hipótesis para la discusión y abrir interrogantes que pueden ser objeto de estudios posteriores.

\section{LA NECESIDAD DE LOS PACTOS EDUCATIVOS}

Reconocer que las estrategias de acción educativa deben ser diseñadas a través de la participación de todos los actores sociales ya puede ser considerado un lugar común en la literatura reciente sobre políticas educativas. Dos argumentos son los más frecuentemente mencionados al respecto. En primer lugar, la necesidad de la participación y el acuerdo se deriva de la propia complejidad del proceso educativo, donde la responsabilidad y la autoridad tienen que ser compartidas por todos los actores sociales que participan de ella. En segundo lugar, la continuidad en la aplicación de las estrategias de transformación ha sido reconocida como una de sus condiciones de éxito y para que exista continuidad, al menos en contextos democráticos, es necesaria la existencia de un nivel básico de acuerdo y de compromiso de todos los actores en su aplicación.

Desde este punto de vista, la última década del siglo XX se inició en un clima de fuerte optimismo acerca de la necesidad de definir estrategias educativas a través de la participación y el consenso de los diferentes actores. Una de las expresiones más significativas de dicho optimismo fue la Conferencia Internacional Educación para Todos, realizada en Jomtiem (Thailandia), en marzo de 1990, convocada por los principales organismos de cooperación internacional (UNESCO, UNICEF, Banco Mundial, PNUD). Uno de los postulados básicos de dicha conferencia fue la necesidad de establecer nuevas alianzas en favor de la educación (WORLD CONFERENCE ON EDUCATION FOR ALL, 1990) y el factor clave del optimismo acerca de las posibilidades de construirlas fue la constatación según la cual - en los nuevos escenarios sociales que surgían a partir de la expansión de las nuevas tecnologías y de la democracia política - el conocimiento constituye la variable central tanto desde el punto de vista de la competitividad económica como del desempeño ciudadano y de la equidad social.

De acuerdo al clima que reinaba en esos momentos tanto en los ámbitos políticos como académicos y productivos, la centralidad del conocimiento en la sociedad daría bases objetivas a las alianzas entre sectores interesados en la competitividad económica, en la ciudadanía 
política o en la equidad social. Estas ideas mantienen su vigencia y podemos ver reiterados ejemplos de ello en los países avanzados y en los países emergentes en proceso de desarrollo y rápido crecimiento (Ver recuadro 1).

\section{Recuadro I. Pacto educativo en los EEUU}

Provistos con uno de los análisis de datos más completos y actualizados que existen hoy en dia, dirigentes empresarios de máximo nivel y líderes de educación superior dijeron que los mediocres resultados obtenidos en ciencias y matemáticas colocaron al pais en un grave peligro de perder su ventaja competitiva en el mercado global.

Los datos - presentados en un nuevo informe por el Business-Higher Education Forum (BHEF) - forman parte de un esfuerzo conjunto de las comunidades empresarias y de educación superior, a fin de analizar objetivamente la información más reciente y actualizada sobre los resultados obtenidos en Norteamérica en ciencias y matemáticas.

"Los datos más recientes sobre el rendimiento de los alumnos estadounidenses en matemáticas y ciencias son objeto de intensa preocupación”, dijo William H. Swanson, Presidente y CEO de Raytheon Company y co-presidente de la Iniciativa sobre Educación en Ciencias y Matemáticas de la BHEF. "La tecnologia es la parte vital de nuestro país porque la innovación crea prosperidad, trabajos buenos y serios para nuestra diversa y creciente población activa. Si no invertimos ni mejoramos el rendimiento de los alumnos de matemáticas y ciencias, esto tendrá serias implicancias en la comunidad empresaria, la economía de los Estados Unidos y nuestra calidad de vida."

El estudio "Compromiso con el Futuro de Norteamérica: cómo responder a la crisis educativa en ciencias y matemáticas”, advierte que, si continúan las tendencias actuales, los Estados Unidos perderán su preeminencia en ciencia y tecnologia y su posición de liderazgo en innovación. Entre los datos clave citados en el informe figuran los siguientes:

- El Programa 2004 para la Evaluación de Estudiantes Internacionales demostró que las habilidades para resolver problemas de alumnos norteamericanos de $10^{\circ}$ grado son significativamente inferiores que las de sus pares en 25 países.

- Aun cuando los Estados Unidos están en medio de un boom de matricula universitaria, los indices de matricula en países con economías y poblaciones emergentes están aumentando en forma aún más rápida a un ritmo extraordinario, similar al que tenía Estados Unidos después de la Segunda Guerra Mundial.

- El Ministerio de Trabajo de los Estados Unidos predice que, en la década que finalizará en el 2008, los empleos que requieren una capacitación cientifico-técnica y de ingeniería aumentarán en un 51 por ciento, un ritmo cuatro veces mayor al crecimiento de empleos en general. Además, en el 2008 habrá unos seis millones de ofertas laborales para científicos, ingenieros y técnicos. 
"La investigación siempre ha apuntado a los docentes como la clave para mejorar el rendimiento de los alumnos", dijo Dennis Smith, Presidente Emérito de la Universidad de Nebraska y Co-Presidente de la Iniciativa BHEF. "Para crear un cuerpo docente altamente calificado, las instituciones de educación superior deben elevar la preparación de docentes de matemáticas y ciencias a un rol central en la misión de sus instituciones".

El informe del BHEF recomienda tácticas cohesivas a largo plazo para paliar los problemas sistemáticos y urgentes tales como la escasez de docentes. Más específicamente, el informe desafía a líderes empresarios, educativos y especialistas en políticas a comprometerse con roles nuevos y de colaboración que fomentarán el desarrollo de sistemas estatales perfectos de educación - sistemas que se extenderán desde el jardín de infantes hasta la educación media y el lugar de trabajo.

"Una de las herramientas más importantes recomendadas por el informe BHEF es la creación de consejos P-16 en el ámbito estatal que incluyan lideres empresarios, del área de educación y gubernamentales. Estos consejos, guiados por el considerable trabajo existente relacionado con el contenido de los cursos, el plan de estudios y las normas, servirán de soporte a los distritos escolares para implementar estrategias innovadoras a fin de mejorar eta rendimiento del alumnado en matemáticas y ciencias”, dijo Warren Baker, Presidente de la California Polytechnic State University y el tercer co-presidente de la Iniciativa BHEF.

El informe completo, "A Commitment to America's Future" (Compromiso con el Futuro de Norteamérica) puede descargarse de la página Web: http://www.bhef.com.

El Business-Higher Education Forum (BHEF) es u a organización sin fines de lucro de líderes provenientes de empresas y universidades, museos y fundaciones. El propósito del grupo es unirse para analizar temas de importancia nacional y, cuando correspondiese, hablar con una sola voz por medio de la publicación de informes, libros blancos y posiciones respecto de políticas, y a través del patrocinio de mesas redondas con funcionarios públicos electos, representantes de las comunidades corporativa y académica y con el público en general.

\section{Pactos educativos y estrategias de desarrollo}

Si bien estas nuevas ideas acerca del papel del conocimiento en el desarrollo social fueron elaboradas fundamentalmente en los países avanzados, se incorporaron rápidamente a las discusiones políticas de los países en desarrollo. Al respecto, es importante recordar que las estrategias de desarrollo vigentes en América Latina hasta los años '90 estuvieron basadas en tres factores principales: la inflación, el endeudamiento externo y la depredación de los recursos naturales. Con estrategias de este tipo, fue posible que algunos países lograran crecer sin equidad social, que otros lograran cierto nivel de equidad pero sacrificando su crecimiento y que otros, por fin, ni crecieran ni garantizaran equidad social (FAJNZYLBER, 1989). Lo peculiar del proceso de desarrollo basado en estos factores fue que ningún país 
logró garantizar los dos objetivos: crecer con equidad social. En este contexto, la educación no podía ser objeto de alianzas ni de pactos porque estaba débilmente conectada con los requerimientos productivos (por el escaso uso del progreso técnico como factor de producción) y con los requerimientos políticos (por la debilidad de la inserción ciudadana en los procesos democráticos).

No es éste el lugar para un análisis histórico detallado del proceso de desarrollo latinoamericano, pero lo cierto es que este modelo en todas sus variantes hizo crisis en la década de los años 80-90, dando lugar a lo que la CEPAL denominó la "década perdida". Dicha crisis abrió las puertas a la adopción de nuevos esquemas conceptuales, que fueron incorporados al debate regional con particular intensidad. Desde este punto de vista, lo peculiar del aporte de los países en desarrollo al debate sobre el crecimiento económico fue el énfasis en que las políticas económicas enfrenten simultáneamente el problema del crecimiento y el de la equidad social. En este "enfoque integrado", la equidad social no puede ser concebida como un factor externo al proceso de crecimiento económico sino como una variable cuyo comportamiento tiene significativos efectos productivos e institucionales y sin cuya consideración es imposible explicar y garantizar el carácter sostenido de los procesos de crecimiento económico (CEPAL, 1992).

La variable que permite articular y compatibilizar los objetivos de crecimiento económico y de equidad social es el progreso técnico o, dicho en otros términos, información y conocimiento. Crecimiento sin progreso técnico implica seguir apoyando la competitividad en la disminución del salario y/o en la depredación de los recursos naturales. Pero los análisis sobre las perspectivas de la economía internacional indican que estos factores no pueden garantizar procesos de crecimiento sostenido, por dos razones fundamentales:

(i) porque el peso de un bajo costo en la mano de obra es cada vez menor en la explicación de la productividad económica $y$,

(ii) porque las exigencias ambientales se están convirtiendo en un factor de creciente importancia en los procesos de incorporación al comercio internacional.

A la inversa, equidad y sustentabilidad ambiental sin progreso técnico también son metas imposibles de alcanzar, porque implicarían detener el crecimiento económico y provocarían un nivel de aislamiento incompatibles con el grado de desarrollo y de expectativas sociales alcanzados por gran parte de los países de la región.

En síntesis, si los países de América Latina quieren crecer económicamente, en forma sostenida, deben garantizar equidad social y cuidado ambiental. Para ello es crucial incorporar progreso técnico en las actividades productivas, lo cual exigen invertir prioritariamente en educación, en ciencia y en tecnología.

Pero una de las consecuencias más importantes de este enfoque consiste en reconocer que la vinculación entre progreso técnico y educación coloca el problema en el ámbito de la calidad de la educación y no meramente en la cobertura o en los años de estudio. Para que la educación contribuya efectivamente al progreso técnico, en el contexto de la actual revolución científico-técnica, es preciso que produzca logros de aprendizaje en términos de conocimientos, de habilidades o de valores que satisfagan los requerimientos de desempeño en la sociedad. No se trata, en consecuencia, de cualquier tipo de oferta educativa. La aplicación de los nuevos patrones de desarrollo exige la definición de estrategias de cambio educativo orientadas específicamente a la calidad de los resultados de aprendizaje. 


\section{Pactos educativos y democracia}

La argumentación presentada en el punto anterior está basada fundamentalmente en la dinámica del crecimiento económico. Pero la necesidad y la posibilidad de las alianzas por educación también tienen una justificación importante desde la dimensión política. Al respecto, es importante recordar que en el caso particular de América Latina, vivimos desde finales de los años ' 80 en un contexto de despolarización del debate político. La democracia volvió a instalarse en los países de la región después de un largo período de gobiernos autoritarios y de luchas políticas que alcanzaron el máximo nivel del conflicto y de violencia. El colapso de las formas autoritarias de gobierno y el agotamiento de la lógica de la guerra para resolver los conflictos sociales crearon un clima favorable a la adopción de mecanismos de negociación y de acuerdo político para resolver los conflictos de intereses.

En este contexto de optimismo inicial acerca de las posibilidades abiertas por las nuevas perspectivas, las políticas educativas de varios países de la región incorporaron la idea del pacto, del acuerdo, como un componente significativo de las estrategias de cambio. Pero tal como se expresara en un texto escrito a comienzos de la década, "postular la necesidad del consenso implica aceptar que el logro de los objetivos educacionales no puede quedar librado exclusivamente a la dinámica del mercado ni a la capacidad de cada grupo social para competir en dicho mercado. La convocatoria a un consenso educativo nacional adquiere sentido en el marco de una estrategia de desarrollo que persiga objetivos de transformación productiva y equidad social. Solo en el contexto de un acuerdo global sobre la estrategia de desarrollo es posible construir nuevas alianzas e identificar los puntos de coincidencia y los de conflicto entre todos los sectores sociales" (TEDESCO, 1992).

\section{¿PORQUÉ ES TAN DIFICIL?}

Las experiencias llevadas a cabo por varios países en el diseño e implementación de pactos educativos durante los últimos quince años se encargaron de demostrar que superar el mero reconocimiento retórico acerca de la necesidad de concertar las políticas educativas implica enfrentar dificultades muy importantes, algunas de las cuales son específicas de los nuevos escenarios sociales creados por las propias transformaciones productivas y otras provienen de la cultura política de nuestros países y de nuestra época.

\section{Pactos educativos y nuevas desigualdades}

Con respecto a las transformaciones productivas, es posible postular la hipótesis según la cual el origen de las dificultades para concertar políticas educativas radica en la propia centralidad que ocupa actualmente el conocimiento en la estructura social. Para decirlo en pocas palabras en la medida que la información y el conocimiento constituyen cada vez más las variables claves de la distribución del poder, el control de su producción y de su distribución se convierten en el ámbito donde se desarrollan, y se desarrollarán mucho más en el futuro, los conflictos sociales más significativos (TEDESCO, 1995). 
La evolución social más reciente ha permitido apreciar que, contrariamente a los pronósticos de las hipótesis optimistas sobre las potencialidades democráticas de una economía y una sociedad basada en la producción de conocimientos, ${ }^{1}$ las economías productoras de ideas parecen ser más inequitativas que las que fabrican objetos. Tal como lo expresara Cohen (1998), la propensión a excluir a los que no tienen ideas es más fuerte que la. propensión a excluir a los que no tienen riquezas. La lógica que operaría en las economías productoras de conocimientos es la lógica de la calidad total o la del "error 0". En este tipo de funcionamiento, el menor error pone en crisis el conjunto de la cadena de producción, razón por la cual las calificaciones de los trabajadores en todos los puestos de trabajo deben ser muy altas. En esta lógica de funcionamiento, como sostiene Cohen (1998), los mejores se juntan con los mejores y los mediocres con los mediocres. Las nuevas tecnologías exacerban esta tendencia más o menos natural, al favorecer la descentralización, la externalización de actividades y el achatamiento de las pirámides de organización jerárquica de las unidades de producción (COHEN, 1998).

En este nuevo escenario, se modifican las tradicionales formas de segmentación propias del capitalismo industrial y del modelo fordista de organización del trabajo. De acuerdo a los estudios sobre este tema, la segmentación tiende ahora a establecerse entre bloques completos de unidades productivas y no entre sectores al interior de una determinada empresa o sector de producción. La miseria del capitalismo contemporáneo consiste en crear, en el seno mismo de cada grupo social, tensiones que hasta ahora estaban en el ámbito de las rivalidades inter-grupos (COHEN, 1998). Esta dinámica del proceso productivo explica la aparente paradoja a la que se confronta la observación de los procesos sociales contemporáneos, donde se advierte que el nuevo modo de producción se caracteriza por producir más igualdad y más desigualdad simultáneamente. Entre los que se incorporan al proceso productivo tecnológicamente más avanzado, existe mucha más homogeneidad que en el pasado, pero entre ellos y el resto de las personas que se desempeñan en unidades productivas tecnológicamente atrasadas o que son excluidas del proceso productivo, se establecen distancias mucho más significativas. ${ }^{2}$

Este modelo de organización del trabajo tiene consecuencias sobre la dinámica del empleo y los salarios. No es éste el lugar para analizar estos fenómenos, pero hay consenso en reconocer que uno de los resultados más visibles de estos procesos es la concentración del ingreso y el aumento de la desigualdad. Ambos son fenómenos particularmente importantes en América Latina, que se ha transformado en la región más inequitativa del mundo. ${ }^{3}$ La discusión acerca del vínculo entre modernización tecnológica, globalización y desigualdad es una de las discusiones más relevantes en estos momentos. ${ }^{4}$ Pero más allá de las hipótesis que se puedan formular al respecto, lo cierto es que la concertación sobre las políticas educativas en un contexto de este tipo, no puede ser un proceso exento de dificultades y de duros conflictos. Concertar en un contexto donde existe una fuerte tendencia a excluir y a expulsar, exige poner en juego dimensiones distintas a las tradicionales. Desde este punto de vista, apelar a la concertación de políticas educativas no es sólo postular una forma o una metodología de enfrentar el conflicto sino que, en sí mismo, implica un contenido socialmente significativo ya que la forma como se toman decisiones educativas es hoy uno de los debates de fondo que divide posiciones e intereses antagónicos. 
$\mathrm{Al}$ respecto, la experiencia reciente indica que la concertación es rechazada o resistida particularmente desde dos perspectivas. La primera de ellas es la que proviene de los enfoques económicos y políticos de inspiración "neo-liberal”, según los cuales las decisiones educativas no pueden someterse a procesos de concertación ni de negociación política, sino que deben ser dejadas - como el resto de las decisiones sobre distribución de bienes y servicios - a los mecanismos del mercado, donde la lógica del comportamiento ciudadano es reemplazada por la del "cliente". La segunda es la que proviene de los enfoques fundamentalistas autoritarios, según los cuales las decisiones son o deben ser tomadas sólo por los que controlan el manejo del aparato del Estado, excluyendo toda posibilidad de pluralismo y debate.

En síntesis, la diferencia fundamental entre la concertación de políticas educativas y las alternativas del mercado por un lado o del fundamentalismo autoritario por el otro, radica en el papel que se asigne a la dimensión política. Apelar a la concertación implica resguardar la esfera de la política en la toma de decisiones, ya que obliga a cada actor social a discutir y negociar públicamente sus opciones educativas. El mercado, en cambio, suprime la política y deja la toma de decisiones librada al resultado de decisiones individuales en función de intereses y posibilidades particulares y de corto plazo. El fundamentalismo autoritario, a su vez, elimina la política porque deja todo el poder en manos de un sólo actor social. El gran interrogante que abre esta discusión consiste en saber si la política tiene o tendrá la fuerza suficiente para contrarrestar el peso de las tendencias propias de los intereses económicos.

\section{Pactos educativos y cultura política}

Destacar la relevancia de la dimensión política frente a las alternativas fundamentalistas de mercado o autoritarias, abre la discusión sobre las características de la política en el nuevo capitalismo en general y en América Latina en particular. En este sentido, la paradoja del presente es que las mayores demandas que se presentan al comportamiento político están acompañadas por una significativa erosión de las instituciones del Estado para satisfacer las demandas sociales y de los partidos políticos como organizaciones representativas de la ciudadanía. Desde un punto de vista general, el proceso de globalización ha puesto en crisis las instituciones del Estado-Nación y ha provocado la pérdida de capacidad de control democrático sobre un conjunto significativo de decisiones políticas. Ulrich Beck ha analizado la dinámica del poder mundial y llamó la atención sobre el déficit de institucionalidad política capaz de enfrentar los fenómenos propios de la mundialización. ${ }^{5}$ Toda la institucionalidad política está basada en la idea del Estado-Nación y sólo muy precariamente aparecen mecanismos institucionales capaces de articular discusiones de carácter mundial. Esto implica que muchas decisiones se adoptan sin discusión ni procesos de concertación real, porque no existen dispositivos políticos capaces de garantizar procesos democráticos en la toma de decisiones fuera del ámbito del Estado-Nación. Los ámbitos donde se pone de manifiesto este déficit institucional de manera más visible son los económico-financieros y los vinculados con riesgos globales tales como el cuidado del medio ambiente, el terrorismo internacional, el narco-tráfico, etc. Sin embargo, cada vez más aparecen decisiones de fuerte impacto educativo y cultural que no están sujetas a procesos de concertación. En la medida que la producción cultural se industrializa, las decisiones en este campo se asimilan a decisiones 
económicas. Es así como la creciente oferta educativa virtual y las decisiones empresarias sobre software educativo, por ejemplo, están cada vez más lejos de los procesos de concertación de tipo nacional.

En el caso particular de algunos países de América Latina, es preciso recordar que la globalización fue concomitante con la superación del autoritarismo y el retorno al Estado de Derecho. Si bien la heterogeneidad de situaciones nacionales es muy grande, es posible sostener que - salvo casos especiales -, los procesos de retorno a la democracia estuvieron asociados con reformas del Estado que provocaron su creciente incapacidad para responder a las demandas sociales. Con la pérdida de instrumentos por parte del Estado para responder tanto a las nuevas como a las tradicionales demandas, se ha expandido un sentimiento general de escepticismo acerca de la potencialidad de la democracia para resolver los problemas sociales. Uno de los indicadores más importantes de este clima es la desconfianza de la ciudadanía con respecto a las instituciones del Estado, hacia los otros actores sociales y hacia el mismo sistema democrático.

Diversas encuestas y estudios nacionales y regionales dan cuenta de este fenómeno. Recientemente se ha presentado el estudio sobre la democracia en América Latina, realizado en 18 países de la región. Según dicho estudio, el 54.7\% de los consultados preferiría un régimen autoritario si garantizara una mejora económica. Esta expectativa no tiene base empírica, ya que la experiencia de los gobiernos autoritarios no indica que hayan sido más capaces de resolverlos problemas que los gobiernos democráticos. Sin embargo, es importante tomar en cuenta este dato para asumir que el reto principal que deben enfrentar los pactos educativos es superar la mera formulación retórica para traducirse en compromisos para la acción (PNUD, 2004a).

La desconfianza, además, ha alcanzado a las instituciones y a los actores sociales responsables de la transmisión del patrimonio cultural y de la cohesión social. En encuestas a muestras representativas de docentes de varios países latinoamericanos se puede apreciar que los dirigentes políticos son el grupo social sobre el cual los docentes tienen mayores niveles de desconfianza (que alcanzan entre el 70 y el $80 \%$ en todos los países estudiados). ${ }^{6}$ La politización es percibida como negativa en la medida que está asociada a conductas de corrupción y de clientelismo. Pero tampoco gozan de confianza otros actores sociales importantes como los empresarios, los dirigentes sindicales, los jueces, los miembros de las fuerzas armadas, etc.

La falta de confianza aumenta en contextos de extrema pobreza. Casos como los de Bolivia y Haití son una muestra de situaciones donde, por un lado, el acuerdo es absolutamente imprescindible para salir de la situación de crisis y, por el otro, es prácticamente imposible lograr acuerdos mínimos para impulsar procesos concretos de acción. En este sentido, la experiencia de estos países permite apreciar la enorme complejidad que tiene la construcción de procesos de concertación en situaciones de emergencia social. Desde el punto de vista de las condiciones objetivas, en contextos de pobreza todas las demandas son urgentes y nadie acepta ser postergado. Los acuerdos son muy difíciles incluso entre diferentes sectores de la población que viven en condiciones de pobreza. Los conflictos de "pobres contra pobres" constituyen un fenómeno cada vez más frecuente en estos escenarios, donde la escasez de recursos provoca pugnas muy fuertes entre sectores que comparten la condición de desfavorecidos. Pero en estos contextos también es preciso incorporar al análisis la dimensión subjetiva expresada a través de la desconfianza hacia el otro y hacia las 
conductas asociadas a los procesos de desarrollo social y político: asumir riesgos, enfrentar la incertidumbre, etc.. La desconfianza es el producto de experiencias vividas por los diferentes actores sociales, que se expresan a través de determinadas representaciones, estereotipos y prejuicios. Por esta razón, la subjetividad ha comenzado a ser considerada como una dimensión del comportamiento social y político, que debe ser tenida en cuenta para el diseño de dispositivos de procesos de concertación. ${ }^{7}$

\section{Pactos educativos y ausencia de futuro}

Además de la debilidad de las instituciones y de las formas de participación política, las nuevas condiciones sociales generan un clima cultural en el cual se pone de manifiesto una fuerte tendencia a concentrar todo en el presente y en el corto plazo. Los análisis sobre la dimensión temporal en la cultura contemporánea ponen de manifiesto este fenómeno, vinculado a la ruptura con el pasado y la incertidumbre sobre el futuro. La ruptura con el pasado está provocada por el profundo cambio tecnológico, político y cultural. La incertidumbre sobre el futuro proviene del déficit de sentido que caracteriza a nuestra sociedad en estos momentos y los altos grados de riesgo y vulnerabilidad con los cuales percibimos el porvenir.

Tal como lo expresara Richard Sennet (2000) en su análisis sobre las dimensiones subjetivas en los procesos económicos actuales, el lema que parece orientar la economía actual es "nada a largo plazo". Según sus estudios, las características especiales que asume la dimensión temporal en este nuevo capitalismo han creado un conflicto entre carácter y experiencia. La experiencia de un tiempo desarticulado amenaza la capacidad de la gente de consolidar su carácter en narraciones duraderas. Así, por ejemplo, sabemos que una persona medianamente educada cambiará frecuentemente de trabajo y de calificaciones, que una empresa nueva que exige al comienzo un esfuerzo y una adhesión intensivas por parte del personal, cuando sale a cotizar en la Bolsa, los fundadores están habilitados para vender, fusionarse, etc. y dejar de lado a los empleados. Los especialistas en administración de empresas aconsejan a los jóvenes trabajar "afuera" de las empresas. Las consultorías son mejores que enredarse en empleos de larga duración. El desapego, la cooperación superficial son mucho más funcionales que la lealtad y el servicio.

Todas estas conductas generan una idea de ausencia de futuro que erosiona las posibilidades de tomar decisiones sobre cuestiones de importancia crucial tanto para el destino personal como colectivo, que exigen un grado muy alto de reflexividad. Hoy, por ejemplo, los ciudadanos de cualquier sociedad están enfrentados a decisiones sobre aspectos tales como incluir o excluir, manipular genéticamente o no a las futuras generaciones, proteger o no el medio ambiente. Estas son algunas de las opciones sobre las cuales los ciudadanos están (o deberían estar) llamados a decidir y todas estas cuestiones ponen en juego nuestros conocimientos e informaciones pero también nuestros valores. Las decisiones están asociadas a valores de solidaridad y responsabilidad con el otro, así como al manejo de conocimientos científico-técnicos que nos brinden el máximo de seguridad acerca de las consecuencias de nuestras decisiones.

Giddens (1999), Beck (2002) y otros sociólogos contemporáneos han aludido al carácter reflexivo que tiene actualmente el comportamiento ciudadano, en oposición al comportamiento determinado por las fuentes tradicionales de confianza. Según sus análisis, se ha 
producido una significativa erosión de los cuatro ámbitos de confianza propios de las culturas premodernas: el sistema de parentesco, la comunidad local, la cosmología religiosa y la tradición. En las sociedades y culturas modernas, el comportamiento ciudadano se basa mucho más en el conocimiento y en la información, pero la característica principal de estas fuentes de comportamiento es que no garantizan certidumbre. Al contrario, la validez del conocimiento es, por definición, cambiante y transitoria. La confianza y la fiabilidad son, en consecuencia, mucho más dificiles de obtener. A su vez, varios analistas de los actuales procesos de cambio han señalado que una de las características más peculiares del nuevo capitalismo es la concentración en el presente, la ausencia de sentido y el carácter efimero de todos los vínculos sociales. Al respecto, Alain Minc (1994) ya a comienzos de los años '90 sugirió que el nuevo capitalismo se asociaba, en realidad, a una nueva Edad Media, donde había ausencia de sistemas organizados, de todo centro articulador y donde "... las solidaridades serán fluidas y evanescentes". Segun Minc, una sociedad sin actores estructurados, sin lealtades firmes, en una estructura social "caótica", fluida, que hace imposible el consenso. La gestión, el "buen" gobierno, estarían basados ahora más en la imaginación y el riesgo que en la confianza y la cohesión. Más allá de la vaguedad de este planteo, el punto principal a retener es el de la falta de estabilidad en los acuerdos. Un muy bajo grado de estabilidad crearía una situación de caos social mientras que un acuerdo de largo plazo seria poco sustentable por el alto dinamismo de la situación social. Esta situación general adquiere significados específicos en contextos de mucha desigualdad no sólo social sino educativa. En un marco de alta reflexividad, los no-educados ocupan un lugar diferente al que ocupaban en sociedades donde el comportamiento ciudadano se regulaba por alguno de los factores tradicionales. En estos contextos, la erosión de los factores tradicionales no está acompañada por el dominio de la información y el conocimiento. Se destruyen los factores tradicionales pero no aparecen los nuevos, con lo cual se exacerban los riesgos de clientelismo, manipulación o despotismo ilustrado. En este sentido, sabemos que los actuales contextos de pobreza se diferencian de los tradicionales por la ruptura de los vínculos de cohesión y confianza y por la pérdida de capacidad para definir proyectos y para expresar demandas.

Existe una fuerte tendencia a "vivir al día", lo cual genera muy pocas posibilidades de participar activamente en procesos de concertación de políticas públicas.

\section{Pactos educativos y "exceso de demandas"}

Las dificultades para diseñar e implementar pactos educativos derivadas de las características generales del nuevo capitalismo adquieren un sentido específico en contextos como los latinoamericanos, caracterizados por la acumulación de demandas que provienen tanto de las deudas del pasado como de los-nuevos desafios planteados por las exigencias de las transformaciones en la organización del Trabajo, la cultura y el desempeño ciudadano. La relevancia de este nuevo contexto no puede ser subestimada a la hora de explicar los problemas y de definir estrategias de transformación. En pocas palabras, lo que queremos decir es que en el contexto actual de transformación profunda de la sociedad en todos sus niveles, los países de la región deben enfrentar simultáneamente las demandas educativas postergadas de los sectores de menores ingresos y las demandas para satisfacer las nuevas exigencias por parte de los sectores integrados. No estamos, en consecuencia, en una situación donde sólo se expresan las demandas insatisfechas 
de los sectores que no han podido tener acceso a un servicio estable, sino ante demandas que provienen también de los sectores que ya han logrado el acceso y ahora exigen su transformación.

En contextos de este tipo, caracterizados por un exceso de demandas, parece inevitable tomar decisiones donde para satisfacer a algunos se postergan los requerimientos de otros. Como todas las demandas son urgentes y legítimas, ningún sector está dispuesto a postergar las suyas. La pugna por obtener los escasos recursos disponibles asume, de esta manera, características poco racionales desde el punto de vista de los intereses generales y de largo plazo. Los riesgos de esta situación son bien conocidos. El más obvio y visible es que las decisiones acerca de prioridades y de asignación de recursos se tomen en favor de aquellos que tienen mayor capacidad de expresar demandas y de ejercer presión para satisfacerlas. Otro riesgo, menos visible pero real en varios países de la región, es la reacción negativa de los sectores integrados hacia las políticas destinadas a promover mayor acceso y participación de los excluidos. Esta reacción negativa se pone frecuentemente de manifiesto a través de la subestimación de la importancia de estos esfuerzos y de la crítica por su bajo impacto en mejorar la calidad o, desde un punto de vista más estructural, a través del abandono de los circuitos de escolaridad públicos y la expansión de la enseñanza privada, que concentra la utilización de las inversiones educativas de los sectores de más altos recursos.

De esta forma, la distancia entre los objetivos planteados y las decisiones adoptadas tiende a aumentar, dando lugar a un fenómeno de disociación, percibido socialmente, donde los objetivos que se expresan en los discursos no se corresponden con las prácticas reales. ${ }^{9}$

En síntesis, los pactos son necesarios para enfrentar la dinámica neoliberal o fundamentalista, pero al mismo tiempo son más exigentes en términos de articulación entre saber experto y lego, en términos de superación de visiones particularistas y en términos de la significación social de las cuestiones sobre las cuales los ciudadanos son convocados a pactar. Estas dificultades, sin embargo, también incrementan significativamente la necesidad de los pactos. El aspecto mas importante sobre el cual es preciso basar el análisis acerca de cómo superar esas dificultades consiste en reconocer la importancia del aspecto reflexivo, voluntario, conciente, claramente político, que asume la tarea de construir los pactos educativos. En ese sentido y evitando la tentación de un exceso de voluntarismo, pareceria importante señalar algunas características del rol del Estado en estos procesos, tanto desde el punto de vista del contenido de su acción como desde el punto de vista de la representación de los diferentes intereses y sectores sociales.

\section{LOS PACTOS SON POSIBLES}

Postular que los pactos son posibles significa sostener la posibilidad de superar el conjunto de dificultades señaladas en los puntos anteriores. La tarea, obviamente, es de una enorme complejidad. Sin embargo, es importante asumir que los pactos deben ser construidos socialmente $y$, en ese sentido, deben ser considerados como un proceso que puede tener momentos de ritmos más intensos o más lentos, que pueden abarcar más o menos actores y más o menos amplitud desde el punto de vista de los compromisos asumidos. Para analizar el espectro de posibilidades que se abren en este complejo proceso de construcción de los pactos, es necesario observar sus diferentes componentes con el máximo de precisión que nos permite la información disponible. 


\section{¿Quién participa y a quien representa?}

El primer aspecto a considerar en el análisis de la construcción posible de pactos educativos se refiere a la definición de quien participa y a quien representa. En este sentido, los procesos de concertación están directamente vinculados al funcionamiento de las instancias democráticas de la sociedad. $\mathrm{Al}$ respecto, una literatura muy exhaustiva se ha ocupado recientemente de analizar el impacto que tienen las transformaciones sociales, culturales, económicas y tecnológicas sobre el funcionamiento de la democracia. En síntesis, se reconoce que fenómenos tales como los cambios en las identidades profesionales, la erosión de las identidades nacionales, la importancia cada vez mayor de la identificación a través de variables adscriptivas como el género o la etnia o de variables de tipo cultural como la religión o la lengua, están modificando significativamente las modalidades de participación y de representación social clásicas de la democracia política.

Todos estos cambios inciden, obviamente, en los procesos de concertación educativa que, sin embargo, mantienen algunos rasgos específicos que los distinguen de otros procesos de concertación política o económica. En primer lugar, es preciso señalar el fenómeno de multiplicación de los actores que participan en la concertación educativa y la ampliación de aspectos o dimensiones que intervienen en la discusión. Someramente expuesto, hemos pasado de una situación en la cual la discusión educativa se concentraba en tres actores fundamentales: el Estado, la familia y la Iglesia, que discutían dimensiones fundamentalmente político-culturales, a una situación donde se multiplicaron tanto los actores como las dimensiones bajo discusión.

En primer lugar, las discusiones educativas han incorporado activamente a los actores del mundo del trabajo, particularmente los empresarios. El comportamiento de los empresarios frente a la educación está vinculado al rol del conocimiento en el proceso productivo y, en este sentido, cambia según los niveles de desarrollo del país y de las empresas. La experiencia muestra, sin embargo, dos limitaciones importantes en el enfoque con el cual las empresas asumen sus compromisos con la educación. En primer lugar, las empresas están más dispuestas a efectuar acuerdos con instituciones educativas más que con los sistemas educativos globales. En segundo lugar, hay una diferencia importante en la apreciación de la dimensión temporal. Mientras las escuelas y los educadores tienen una dimensión de largo plazo, los empresarios pretenden resultados inmediatos.

Los sindicatos de trabajadores muestran, hasta ahora, menor interés y conciencia de la importancia de lo que está en juego en la distribución y utilización de los conocimientos en el procesos productivo y una hipótesis probable de este fenómeno consiste en sostener que el bajo grado de interés sindical por la educación está vinculado con el debilitamiento general de la cohesión sindical en procesos productivos intensivos en conocimientos.

Otro nuevo actor en el proceso educativo son los medios de comunicación .y sus actores internos. Si bien los medios juegan un papel cada vez más importante en el proceso de socialización de las nuevas generaciones, su participación en los procesos de concertación educativa es muy débil.

Pero también es preciso observar que la mayor apertura a los actores externos al sistema educativo en los procesos de concertación educativa ha provocado una reacción de movilización y aumento del corporativismo de los actores internos, que perciben la participación de otros actores como una pérdida de protagonismo y de reconocimiento al carácter técnico- 
profesional de su trabajo y de la discusión sobre la naturaleza de su trabajo. Los actores internos del sistema educativo enfatizan la mayor relevancia de las dimensiones propiamente pedagógicas de la educación (¿cómo enseñar?), frente a los aspectos "funcionales" (formar la ciudadanía, formar para el trabajo, etc.).

Por último, también es preciso señalar la presencia - particularmente importante en los países en desarrollo pero no exclusivamente en ellos - de otro nuevo actor vinculado al proceso de globalización: los organismos internacionales de financiamiento $\mathrm{Z}$ de cooperacion y de investigación educativa. Estos organismos juegan actualmente un papel muy importante, pero su heterogeneidad no debe ser subestimada. Existen diferencias muy significativas entre el papel que juegan los organismos de financiamiento internacional, los organismos intergubernamentales de asistencia técnica y de consenso político y las organizaciones internacionales no-gubernamentales, ya sean de carácter político o técnico. Todos ellos han adquirido mayor protagonismo en el marco del proceso de globalización, pero sus orientaciones ideológicas, sus modalidades de gestión y de vinculación con los estados nacionales, son muy diferentes. En este aspecto, es muy importante tener en cuenta la posibilidad de pactos internacionales en educación, que superen los meros acuerdos políticos que resultan habitualmente de las conferencias internacionales de educación. Un ejemplo de los nuevos pactos es el que ofrece actualmente la negociación acerca del tema de canje de deuda por educación, donde los gobiernos de Argentina y España están avanzando seriamente en la firma de un acuerdos re este tema.

La multiplicación de actores ha estado asociada a la ampliación del espectro de dimensiones que intervienen en el proceso de concertación educativa. Además de las dimensiones clásicas de tipo político-cultural, donde se negociaban fundamentalmente los contenidos valóricos de la educación, ahora asume gran importancia la dimensión económica (tanto en el sentido educativo como propiamente financiero y presupuestario), y todo lo relativo a la subjetividad de los actores involucrados en el proceso pedagógico (respeto a la identidad cultural, procesos de individualización, etc.)

En este sentido, es preciso reconocer que la representación a través de los partid s políticos no logra cubrir la identidad de los diferentes actores sociales frente a la educación. La debilidad o la parcialidad de la identidad política con respecto a los problemas educativos refuerza el papel de las instancias corporativas internas de participación (docentes, autoridades centrales, locales, etc.), o de aquellas instancias externas con intereses educativos muy específicos (Iglesia, empresarios, etc.). No es casual, por ello, que no exista una asociación clara entre demandas educativas y partidos políticos y que cada uno de ellos, cuando está en el poder, asume las posiciones educativas propias del poder y, cuando pasa a la oposición, asume las posiciones e oposición.

Esta distinción entre la concertación con actores externos y la concertación interna está asociada a los contenidos de los temas a discutir. Mientras con los primeros se negocian y discuten especialmente los objetivos, los contenidos básicos y las orientaciones valorativas, con los segundos se discuten las estrategias, las modalidades de acción y la distribución de recursos.

En este sentido, es importante advertir sobre la necesidad de evitar la disociación entre estos dos niveles. Un riesgo visible en algunos casos latinoamericanos es que dicha disociación provoca un progresivo proceso de corporativización de la participación. En la discusión sobre objetivos existen amplios procesos de consulta y de discusión, donde la participación 
de los actores externos suele ser muy amplia. Fenómenos como el congreso pedagógico de Argentina, el día "D" de la educación en Brasil, la Consulta popular sobre educación en Ecuador, la consulta en República Dominicana, etc., son todos ejemplos de procesos de movilización de la opinión pública que generaron fuertes demandas. Cuando la discusión llega a la fase de definir compromisos y modalidades de acción, la diversidad de actores que participan tiende a disminuir y la participación adquiere un carácter más corporativo.

$\mathrm{Al}$ respecto, las experiencias realizadas confirman que existe una diferencia muy importante entre los acuerdos y consensos que se logran con respecto a los objetivos generales y los acuerdos referidos al proceso de implementación. Obtener consenso en la fase de definición de objetivos resulta relativamente fácil si se lo compara con las enormes dificultades que existen para obtener consensos en el momento de la ejecución. Este fenómeno está asociado con, al menos, dos aspectos diferentes: (i) los acuerdos sobre objetivos se logran a través de procesos mediante los cuales se aumenta cada vez más el grado de generalidad, para evitar que se pongan en la mesa de discusiones los verdaderos problemas y las consecuencias de los cambios sobre los diferentes actores, y (ii) es probable que ninguno de los actores conozca exactamente cuales serán las consecuencias de los cambios y que sólo en la fase de aplicación de las estrategias de cambio, aparecerán las verdaderas magnitudes de las transformaciones diseñadas previamente.

Finalmente, es preciso considerar todo lo que se refiere a quien asume la responsabilidad de convocar a los diferentes actores a que negocien, a que dialoguen y a que concerten. Hay muchos ejemplos de procesos de concertación convocados y coordinados por organizaciones no gubernamentales, por agencias internacionales, por la Iglesia o por una combinación de estas instituciones. Cuando esto sucede es porque ya se ha instalado una crisis profunda en la sociedad y en el aparato del Estado, que es percibido como ausente o como representante de un determinado sector y no de los intereses generales. Si bien la iniciativa en la convocatoria a construir pactos educativos puede ser asumida por diferentes instituciones según los contextos y los momentos históricos, el papel y la responsabilidad del Estado en este proceso es insustituible. El Estado es responsable de convocar, pero también tiene un papel importante en términos de representación. Al respecto y si bien el Estado asume los intereses generales, su responsabilidad principal es representar a los excluidos. Sólo el Estado puede hablar por los que están afuera y no están representados a través de organismos corporativos.

\section{Los pactos locales}

El proceso de globalización re-define el significado que asumen en los ámbitos locales todas las dimensiones asociadas a este espacio de participación. También sobre este tema la literatura es muy abundante y existen evidencias que muestran que el ámbito local puede ser tanto un espacio donde se apoyen los fundamentalismos autoritarios de carácter comunitarista como, a la inversa, escenarios donde la concertación, las alianzas y los pactos pueden tener mayores posibilidades fácticas. En este último sentido, es posible evocar los ejemplos de pactos diálogos regionales de educación, así como algunas experiencias de proyectos educativos basados en la idea de comunidad de aprendizaje o de padrinazgo de escuelas por parte de empresas u otras instituciones.

Los pactos educativos de nivel local se han visto estimulados por varias razones. Desde el punto de vista administrativo, los pactos locales han adquirido relevancia en el marco de 
los procesos de descentralización, que en algunos casos transfirieron responsabilidades a los municipios. La experiencia más interesante en el contexto latinoamericano es la de los "Diálogos ciudadanos por la calidad de la educación', que se llevan a cabo en Chile, impulsados por la administración central. En estos diálogos participan profesores, alumnos, académicos, representantes del gobierno regional, padres y madres de los alumnos. Los diálogos se desarrollan en dos modalidades: presencial y vía Internet. Ya se han realizado cinco encuentros regionales presenciales y el diálogo vía Internet se desarrolla a través del sitio Web http://dialogo. minudec.cl

Pero otra fuente que justifica los pactos locales es el carácter integral de las estrategias destinadas a promover el desarrollo comunitario. En muchos lugares se advierte ya sea la necesidad de diseñar planes estratégicos de desarrollo local o planes integrales de acción, que promuevan acciones coordinadas entre distintos sectores de la administración (educación, salud, empleo, vivienda, etc.) y entre diferentes actores sociales (docentes, empresarios, Iglesia, gobierno local, etc.) (NEIROTTI; POGGI, 2004). Sobre la asociación entre empresas y escuelas hay una abundante literatura y experiencias diversas, tanto en los países centrales como en la periferia. $\mathrm{Al}$ respecto, ya hace varios años la $\mathrm{OCDE}$ publicó un libro sobre experiencias de acuerdos entre escuelas y empresas (CERI, 1995). Allí se puede apreciar tanto las principales motivaciones para los acuerdos entre empresas e instituciones educativas, como los límites de estos acuerdos. En primer lugar, las empresas están más dispuestas a efectuar acuerdos con instituciones que con los sistemas educativos globales. En segundo lugar, hay una diferencia importante con respecto al manejo de la dimensión temporal. Mientras las escuelas y los educadores tienen una dimensión de largo plazo, los empresarios pretenden resultados inmediatos.

\section{La dimensión institucional: la alianza entre escuela y familia}

La discusión sobre los vínculos entre escuela y familia o entre docentes y padres es tan larga como la historia de la propia escuela. Sus contenidos, sin embargo, cambian significativamente a partir de las transformaciones que tienen lugar tanto en la familia como en las funciones sociales de la escuela. Sin entrar en un análisis histórico, digamos que en la actualidad nadie puede negar que la participación de las familias es fundamental para el éxito escolar de los alumnos, que las familias deben expresar sus demandas educativas y deben estar informadas de las actividades escolares y que la participación en algunos aspectos de la gestión del centro escolar puede contribuir a aumentar la eficacia y a mejorar los resultados. Pero ¡cuál es el límite o, mejor dicho, cuál es el criterio que define la legitimidad o la ilegitimidad de la participación familiar?. El límite está dado por el carácter público y profesional del trabajo de la escuela.

El carácter público de los centros escolares supone que la definición de los contenidos que ellos transmiten no puede ser el mero reflejo de las demandas de cada actor particular. Los contenidos educativos son la expresión de acuerdos sociales, de compromisos colectivos, asumidos cada vez más reflexivamente. Dichos acuerdos garantizan la cohesión social que permite que podamos vivir juntos. En sociedades democráticas como las nuestras es ilegítimo aceptar, por ejemplo, que una familia demande que sus hijos sean educados en valores de fanatismo e intolerancia. De la misma manera, me atrevería a decir que es socialmente 
ilegítimo que las familias pretendan educar a sus hijos al margen de las instituciones públicas donde la convivencia con los otros es un aspecto fundamental del aprendizaje. Esta tendencia ya se expresa en algunos países (particularmente los EEUU) donde un numero significativo de niños y niñas se educan en sus casas a través de Internet.

El carácter profesional del trabajo de la escuela se define por la eficiencia y la eficacia para lograr que todos los alumnos tengan acceso al máximo nivel de logros de aprendizaje que permiten sus capacidades. Para alcanzar este objetivo, el personal que se desempeña en los centros escolares debe estar dotado de un saber profesional especializado, compuesto por conocimientos técnicos y condiciones subjetivas tales como la confianza en la capacidad de aprendizaje de todos los alumnos, compromiso afectivo, etc.. Los métodos de enseñanza, las formas de organizar las actividades escolares, los métodos de evaluación, etc., exigen personal profesionalmente apto y cada vez más competente para enfrentar los desafios que presenta el objetivo democrático de garantizar altos logros de aprendizaje para todos. Desde este punto de vista, la participación de las familias está limitada por el conocimiento profesional que define el ejercicio de la profesión docente.

Pero para que la escuela y los docentes puedan reivindicar esos límites tienen que ser efectivamente los representantes del mensaje socializador público y los poseedores del saber técnico profesional que garantice buenos resultados. No es posible dejar a las familias afuera de la escuela para defender intereses privados corporativos o para defender la falta de competencia para resolver los problemas. Sobre estas bases y en el marco de modalidades de gestión que otorguen niveles adecuados de autonomía a las escuelas, es posible pensar en acuerdos entre la escuela y las familias, que pueden adquirir modalidades más o menos formales, que van desde la firma de "contratos" hasta la participación en la gestión de algunos aspectos de la vida escolar.

\section{Pactos educativos y pactos sociales}

Después de todo este análisis, corresponde preguntarse si efectivamente los pactos educativos son posibles. Aunque pueda parecer excesivamente voluntarista, el argumento final de este análisis consiste en reconocer que si algo es considerado socialmente necesario, tiene que ser posible. Obviamente, este carácter de necesario y posible está asociado a un proyecto social y político. El pacto no es un instrumento válido en sí mismo, sino que constituye un procedimiento consistente con un proyecto social basado en la idea de construir una sociedad equitativa y dinámica. En ese contexto, por lo tanto, concertar políticas educativas es parte de un proceso más general de fortalecimiento de la ciudadanía y de construcción de un orden político democrático. Desde esta perspectiva filosófico-social, los procesos de concertación democrática son una forma de ejercicio de la solidaridad conciente y reflexiva que exigen las nuevas estructuras sociales. Mientras en el capitalismo industrial existía lo que se concebía como "solidaridad orgánica", es decir una solidaridad semejante a la que existe entre las diferentes partes de un organismo donde no hay una decisión voluntaria de ser solidario, en el nuevo capitalismo, en cambio, los niveles de solidaridad orgánica disminuyen y para vivir juntos será necesario querer vivir juntos, será necesario adherir a un proyecto necesariamente político que se proponga lograr la inclusión de los excluidos, que se proponga garantizar igualdad de oportunidades a todos. La solidaridad reflexiva exige un fuerte 
sentido de pertenencia colectiva, a partir del cual es posible aceptar la idea de la redistribución directa de los bienes. La educación juega, en este sentido, un doble papel: es objeto de redistribución y, al mismo tiempo, es el instrumento para formar los valores de solidaridad que permitan tomar la decisión de redistribuir.

Pero en el contexto latinoamericano, es necesario no perder de vista que u educativo debe ser percibido y asumido como un módulo importante de un pacto más amplio de lo que recientemente la CEPAL ha denominado el "pacto de cohesión social". Sin equidad y sin cohesión social, el crecimiento económico será insostenible y las crisis de gobernabilidad provocarán niveles de inestabilidad política incompatibles con los requerimientos del crecimiento y del ejercicio de la democracia. Colocar los pactos educativos en el marco de la construcción de pactos de cohesión social supone reconocer que intervenir en las dimensiones económicas, particularmente en la distribución del ingreso, tiene un efecto educativo muy relevante. En este sentido es posible recuperar todo lo que se refiere a mejorar las condiciones de educabilidad de los alumnos como condición necesaria para que las estrategias pedagógicas sean exitosas.

\section{LA TENSIÓN ENTRE PACTOY TOMA DE DECISIONES}

Por último, quisiéramos referirnos a un aspecto que suele ser percibido como una contradicción, particularmente entre los responsables de la toma de decisiones. Los pactos y los procesos de concertación entran en tensión con la urgencia que requiere la solución de los problemas. En este sentido, parece importante referirse a tres grandes ejes de tensiones que están presentes en estos procesos.

El primer eje se refiere a la tensión que existe entre el determinismo estructural por un lado y el voluntarismo de los gestores por el otro. El análisis sistémico suele ser muy duro a la hora de marcar obstáculos, límites o, peor aun, la imposibilidad de modificar determinadas situaciones. El análisis de algunas tendencias provocadas por el funcionamiento de los modelos de desarrollo social - tales como el aumento de la desigualdad, de la polarización y de la exclusión social - genera fuertes sentimientos de escepticismo o, como una variante de este escepticismo, la idea según la cual hay que esperar que llegue el cambio estructural para luego iniciar el cambio educativo.

La contrapartida de este escepticismo es el voluntarismo con el cual los gestores de los procesos de cambio educativo pretenden enfrentar los obstáculos de la realidad. Es cierto que vivimos un momento histórico muy particular, donde las estructuras se están modificando y donde existe un margen más amplio que en el pasado para orientar el sentido de los cambios. Pero el espacio para la voluntad no es infinito. Sólo si somos conscientes de los límites de la realidad, de los intereses y de las posibilidades con las cuales actúan los diferentes actores, será posible que la voluntad no se transforme en mero voluntarismo. A la inversa, el conocimiento de la realidad y de las posibilidades no puede desconocer el factor de cambio que existe en el comportamiento de los actores sociales. Sin este reconocimiento, el determinismo se transforma en pasividad y aceptación del orden existente. Manejar esta tensión es uno de los aspectos más difíciles de toda persona que debe asumir la responsabilidad de tomar decisiones. 
El segundo eje se refiere a la tensión entre la competencia técnica y el compromiso político. La competencia técnica sin compromiso político es mera tecnocracia. El compromiso político sin competencia técnica es clientelismo, demagogia o simplemente una militancia ineficiente. Pero el contacto directo con protagonistas de los procesos de transformación educativa permite apreciar que esta separación tiende a desaparecer. Por un lado, los factores emocionales, personales, actitudinales - tradicionalmente asociados al compromiso político - constituyen hoy un componente técnico fundamental para el éxito de los procesos de gestión. El compromiso político es hoy una característica fundamental de la competencia técnica. Pero, a la inversa, si el compromiso político no va acompañado por un alto nivel de competencia técnica, de conocimientos actualizados de todos los instrumento que nos brinda el desarrollo tecnológico, no logra superar su carácter meramente retórico, discursivo.

Si bien el pluralismo constituye el principio fundamental que en un contexto democrático orienta la dinámica de la participación política, no cabe duda que ese pluralismo se enmarca en un compromiso político muy firme con la equidad social. Nuestra región tiene esta deuda pendiente. Tenemos el vergonzoso primer puesto en términos de inequidad social a nivel internacional $y$, en este sentido, el compromiso de los educadores es trabajar a favor de la reducción de estos niveles de inequidad y desigualdad social, que constituyen no sólo un problema social, económico y político, sino también un fuerte llamado ético. En definitiva, no podemos darnos el lujo de ser ineficientes.

El tercer eje es el que se refiere a la tensión que existe entre la negociación, la concertación, la búsqueda de consensos y la necesidad de actuar, de pasar a la fase de la ejecución de las acciones. Los argumentos a favor de los consensos, los acuerdos y las negociaciones son bien conocidos por todos. Pero la urgencia, la necesidad de actuar para resolver los problemas, suele también ejercer una presión muy fuerte para acortar los tiempos de las negociaciones. Negociaciones interminables anulan el valor de la participación. Ejecuciones rápidas suelen ser efímeras porque no gozan del acuerdo necesario para su permanencia ni de la garantía de haber sido elaboradas con la reflexión suficiente para que sean las mejores soluciones. Una de las competencias más importantes de la administración de políticas públicas es, precisamente, la capacidad para manejar esta tensión y definir el momento más oportuno para traducir la negociación en acciones concretas. No hay separación rígida entre ambos momentos. La negociación es la base de las decisiones para la acción y los efectos de la acción alimentan el proceso de negociación.

Argentina, al igual que otros países latinoamericanos, está ante una nueva oportunidad para enfrentar los retos que plantea la construcción de sociedades justas, cohesionadas, donde sea posible vivir juntos y todos tengan posibilidades de desarrollarse como personas dignas y felices. Los pactos educativos son una herramienta para el logro de este objetivo. La responsabilidad de no perder esta nueva oportunidad está en las manos de sus elites dirigentes. 


\section{Notas}

1 Una versión muy popular de estas hipótesis optimistas puede verse en Alvin Toffler, El Cambio del Poder. Barcelona: Plaza y Janés, 1990.

2 La literatura al respecto es muy abundante. Puede verse el ya clásico libro de Manuel Castells. $\mathrm{La}$ era de la información. Economía, sociedad y cultura. 3 vols., Madrid: Alianza, 1997. También Robert Castel. Metamorfosis de la cuestión social. Una crónica del salariado. Buenos Aires: Paidós, 1997. Un resumen desde la dimensión educativa puede verse en Juan Carlos Tedesco. Educar en la sociedad del conocimiento. Buenos Aires: Fondo de Cultura Económica, 2002.

3 Según datos procesados por el PNUD, el coeficiente de Gini para el conjunto de los países de la región creció entre 1990 y 2003 del 0.554 al 0.566, mientras que el promedio mundial es de 0.381 .

4 Ver, por ejemplo, los trabajos incluidos en Fernando Calderón (Coord.). ¿Es sostenible la globalización en América Latina?. Debates con Manuel Castels. 2 vols. Santiago de Chile: Fondo de Cultura Económica, 2003, especialmente el texto de A. Ortuño y C. Pine, "Globalización, desigualdad y reformas en la América Latina de los años noventa".

5 Un aporte muy importante a esta discusión puede verse en Ulrich Beck. Pouvoir et contre-pouvoir à 1 'ère de la mondialisation. Paris: Aubier, 2002.

6 El IIPE-UNESCO Buenos Aires efectuó entre el año 2000 y 2001 una encuesta a muestras representativas de maestros y profesores de cuatro países: Argentina, Brasil, Perú y Uruguay. El grupo social que registra mayor nivel de desconfianza entre los docentes de los cuatro países en "los políticos": Argentina 87\%, Brasil 85\%, Perú 76\% y Uruguay 67\%. En Perú y Argentina siguen en el ranking de mayor desconfianza los funcionarios públicos (84 y 62\% respectivamente), los sindicalistas (sólo en el caso de Argentina con 81\%) y los magistrados (65 y 67\%). En el otro extremo se observa que los docentes confian masivamente en ellos mismos y luego en los periodistas y en los sacerdotes.

7 Un muy interesante análisis de la situación de Bolivia con respecto a las condiciones subjetivas para enfrentar los procesos de desarrollo puede verse en PNUD. Interculturalismo y globalización; La Bolivia posible. Informe de Desarrollo Humano 2004. La Paz, $2004 \mathrm{~b}$.

8 Alain Minc. La nueva Edad Media. Madrid, Temas de Hoy, 1994. Sobre la concentración en el presente, es importante ver el análisis de Zaki Laidi. Le sacre du présent. Paris, Flammarion, 2000.

9 Este fenómeno - que caracteriza no sólo a las políticas educativas sino al conjunto de las políticas públicas - está en la base de la debilidad de los procesos democráticos en la región La discusión de este tema está volviendo a ser de actualidad. José Nun, Democracia, igobierno del pueblo o gobierno de los politicos?. Buenos Aires, Fondo de Cultura Económica, 2000.

\section{Referencias}

BECK, Ulrich. Pouvoir et contre-pouvoir à l'ère de la mondialisation. Paris: Aubier, 2002.

BRASLAVSKY, Cecilia. "La concertación como estrategia de Reforma Educativa y del Estado". In: FLACSO - Fundación Concretar. ¿Es posible concertar las políticas educativas?. La concertación de políticas educativas en Argentina y América latina. Buenos Aires: Miño y Dávila Editores, 1995.

CALDERÓN, Fernando (Coord.). ¿Es sostenible la globalización en América Latina?. Debates con Manuel Castells. 2 vols. Santiago de Chile: Fondo de Cultura Económica, 2003.

CASSASUS, Juan. “Concertación y alianzas en Educación”. In: FLACSO - Fundación Concretar. ¿Es posible concertar las políticas educativas?. La concertación de políticas educativas en Argentina y América Latina. Buenos Aires: Miño y Dávila Editores, 1995. 
CASTEL, Roben. Metamorfosis de la cuestión social. Una crónica del salariado. Buenos Aires: Paidós, 1997.

CASTELLS, Manuel. La era de la información. Economía, sociedad y cultura. 3 vols., Madrid: Alianza, 1997.

CEPAL. Equidad y transformación productiva: un enfoque integrado. Santiago de Chile, 1992.

CERI. Schools and business: a new partnership. Paris: OCDE, 1995.

COHEN, David. Riqueza del mundo, pobreza de las naciones. Buenos Aires: Fondo de Cultura Económica, 1998.

FAJNZYLBER, Fernando. Industrialización en América Latina: de la "caja negra" al casillero vacío. Santiago: Cuadernos de la CEPAL, 1989.

FLACSO - Fundación Concretar. ¿Es posible concertar las políticas educativas?. La concertación de políticas educativas en Argentina y América latina. Buenos Aires: Miño y Dávila Editores, 1995.

GIDDENS, Anthony. La tercera vía: la renovación de la socialdemocracia. Madrid: Taurus, 1999.

LAIIDI, Zaki. Le sacre du présent. Paris: Flammarion, 2000.

MINC, Alain. La nueva Edad Media. Madrid: Temas de Hoy, 1994.

NEIROTTI, N.; POGGI, M. Alianzas e innovaciones en proyectos de desarrollo educativo local. Buenos Aires: IIPE - Unesco, 2004.

PNUD. La democracia en América Latina. Hacia una democracia de ciudadanas y ciudadanos. 2004a.

La Paz, 2004b.

Interculturalismo y globalización. La Bolivia posible. Informe de Desarrollo Humano 2004.

SENNETT, Richard. La corrosión del carácter. Las consecuencias personales del trabajo en el nuevo capitalismo. Barcelona: Anagrama, 2000.

TEDESCO, Juan Carlos. "Nuevas estrategias de cambio educativo en América Latina”, Boletín del Proyecto Principal de Educación en América Latina y el Caribe, n. 28, ago. 1992.

. El nuevo pacto educativo: competitividad y ciudadanía en la sociedad moderna. Madrid: AlaudaAnaya, 1995.

- Educar en la sociedad del conocimiento. Buenos Aires: Fondo de Cultura Económica, 2002. . “Porqué son tan dificiles los pactos educativos?”, Revista Iberoamericana de Educación. Madrid. OEI, n. 34, enero-abril 2004.

TOFFLER, Alvin. El cambio del poder. Barcelona: Plaza y Janés, 1990.

WORLD CONFERENCE ON EDUCATION FOR ALL. Meeting Basic Learning Needs: A Mission for the 1990's. Background Document. Jomtien, Thailand, 5-9 march 1990. 


\section{Pactos educativos: difíceis, mas necessários \\ Resumo}

Neste texto, é abordado um dos temas mais importantes dos programas de educação contemporâneos: os pactos educativos. Estes podem ser considerados um produto deliberado das negociações entre os diferentes atores sociais, para enfrentar as tendências à fragmentação, à ruptura e à exclusão, tanto das classes mais pobres da população como das classes mais privilegiadas da estrutura social. Tal documento é dividido em função de três palavras: a necessidade de construir pactos que garantam a continuidade das políticas educativas; a dificuldade em fazê-los; a possibilidade de atingir este objetivo: tornar possivel a construção de sociedades justas e coesas.

Palavras-chave: Pacto educativo. Pacto social. Aliança educativa. Inovação de projetos educativos. Concertação educativa. Políticas educativas.

\section{Educative agreements: difficult but necessary}

\section{Abstract}

In this document one of the most important themes in the contemporary educational agenda is dealt with: educative agreements. These can consider the deliberate product of the negotiation between the different social actors in order to face the tendency of fragmentation, rupture and exclusion both of the poorer sectors of the population and of the sectors which occupy the top of the social structure. The document is divided according to three key words: the need to build agreements which guarantee the continuity of the educational policies; the difficulty to achieve that; the possibility to reach that objective; implement the construction of just and coesive societies.

Keywords: Educative agreement. Social agreement. Educative alliance. Innovation in educational projects. Educative concert. Educative policies.

\section{Pactes éducatifs : difficiles, mais nécessaires}

Résumé

Dans ce document un des plus importants thème dans l'agenda éducationnel contemporain est traité: les pactes éducatifs. Ceux-ci peuvent considérer le produit délibéré de la négotiation entre les différents acteurs sociaux afin de faire face à la tendence de fragmentation, rupture et exclusion autant des secteurs pauvres de la population que les secteurs qui ocuppent le dôme de la structure sociale. Le document est divisé selon trois mots clefs : le besoin de construire des pactes qui garantissent la continuité des politiques éducationnelles; la difficulté d'atteindre cela; la possibilité d'atteindre cet objectif : implémenter la construction de sociétés justes et coésives.

Mots clefs : Pactes éducatifs. Pactes sociaux. Alliances éducatives. Innovation dans les projets éducatifs. Concert éducatif. Politiques éducatives.

Recebido: 30.08 .2005

Aceito: 29.09 .2006 
